\title{
HIPPARCOS SUBDWARFS AND GLOBULAR CLUSTER AGES: TOWARDS RELIABLE ABSOLUTE AGES
}

\author{
F. PONT AND M. MAYOR \\ Geneva Observatory, Sauverny, Switzerland \\ AND \\ C. TURON \\ Observatoire de Paris, Meudon, France
}

\begin{abstract}
.
The maximum age of galactic globular clusters provides the best observational constraint on the minimum age of the Universe. One of the main "missing link" in the globular cluster age determination has been the lack of a precise calibration, with local subdwarfs, of the position of the subdwarf sequence at different $[\mathrm{Fe} / \mathrm{H}]$.

Hipparcos data may change this situation. As many precise parallaxes become available for local subdwarfs, the distance to globular clusters can be estimated directly from ZAMS fitting to the subdwarf locus. The ages can then be inferred from the turnoff luminosity (a robust prediction of stellar evolution models), rather than using secondary indicators such as Horizontal-Branch position, or indicators depending on the uncertain colour scale such as turnoff colour.

Combining Hipparcos parallaxes with $[\mathrm{Fe} / \mathrm{H}]$ values determined with the CORAVEL spectrometer, we are studying the position of the subdwarfs in the Colour-Magnitude Diagram from a sample of more than 900 subdwarf candidates. Preliminary results are presented here. It is shown that the distances of many subdwarfs had been underestimated in previous studies, mainly because a large fraction of them is in fact evolved off the main sequence into the turnoff or the subgiant branch.
\end{abstract}




\section{The Hipparcos Subdwarfs}

We are using parallax data for more than 900 metal-deficient stars measured by the Hipparcos astrometric satellite. This sample is the reunion of two different groups: 332 stars previously known or suspected to be subdwarfs, predominantly F stars, and about 600 stars shown by the Hipparcos parallax data to be situated well below the Main Sequence on the ColourMagnitude Diagram (CMD).

\section{The "Historical" Subdwarfs}

Before the Hipparcos data, less than a dozen subdwarfs possessed wellknown parallaxes. They are included in our sample, and it appears at once (Fig. 2) that most of the previous parallax estimates were inaccurate. Distances were in fact systematically underestimated, often by a large amount.

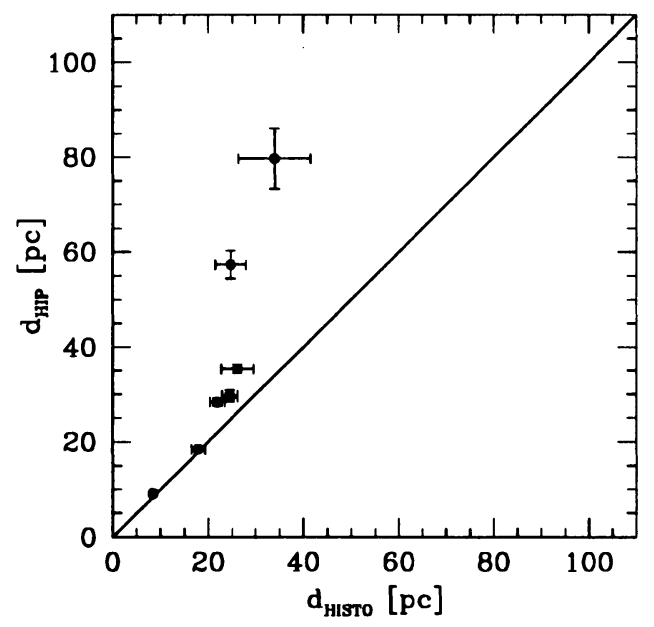

Figure $1 . \quad$ Distance for seven "historical" subdwarfs from the literature (Zinn\&West 1981) and from Hipparcos. Most distances were importantly underestimated.

\section{3. $[\mathrm{Fe} / \mathrm{H}]$ determination with CORAVEL}

The CORAVEL radial velocity spectrometer has been known for a long time to permit precise estimates of the metallicity of dwarf stars via the surface of the cross-correlation function. The cross-correlation function surface, $\mathbf{W}$, representing the average surface of more than 1'500 weak metallic lines, is a sensitive function of $[\mathrm{Fe} / \mathrm{H}]$ and of temperature. As early as 1980 a first calibration was made (Mayor 1980). But only recently have both precise, homogeneous metallicity studies of dwarfs (Edvardsson et al. 1993) and large CORAVEL samples (F-G dwarfs survey, radial velocity survey of Hipparcos stars, cluster data) become available that provide an accurate calibration of the method for $\mathrm{F}$ to $\mathrm{K}$ dwarfs. 
The calibration is made using Edvardsson et al. (1993, E93) for Fdwarfs, and extrapolation based on the Hyades cluster for G-dwarfs, and the Carney et al. (1994, C94) surveys for subdwarfs. The residual dispersion of $[\mathrm{Fe} / \mathrm{H}]$ is 0.08 dex with $\mathrm{E} 93$ and 0.14 dex with $\mathrm{C} 94$. This calibration will be refined as more measurements for subdwarfs and Hyades late-type stars are realized. Corrections for faint stars appear necessary and also have to be calibrated.

The CORAVEL determination is spectroscopic, thus much less sensitive to small anomalies in the colours or binarity than photometric indices, and has the crucial advantages of being available with little observing time in a homogeneous way for large sample of stars, including many of the 60'000 stars already measured with CORAVEL.

The sample of Hipparcos subdwarfs is now being measured with CORAVEL. The aim is a homogeneous sample of $[\mathrm{Fe} / \mathrm{H}]$ determinations for all Hipparcos stars with accurate parallaxes and situated below the solar-metallicity ZAMS, in order to allow an optimal treatment of the biases. In the sample selection, a wide margin has been included below the main sequence, so that contamination by solar-metallicity stars can be controlled closely.

\section{Position of the subdwarfs in the CMD}

With accurate parallaxes and $[\mathrm{Fe} / \mathrm{H}]$ determinations, colour-magnitude diagrams (CMD) can be constructed for different metallicities. Fig. 2 shows for instance the CMD for stars in the -2.4 to -2.0 dex range in $[\mathrm{Fe} / \mathrm{H}]$. For the first time, a field subdwarf sequence is defined.

The important number of evolved stars in the sample is immediately apparent. Indeed such an abundant presence of evolved stars among subdwarfs was not quite expected in recent subdwarfs studies (C94, Ryan \& Norris 1991). C94 for instance estimate the contamination from evolved stars to be about $10 \%$ in their sample. The true value may be much higher, as is made clear in Fig. 3, thus modifying the distance and space velocity estimates. The distances to most F-type subdwarfs were consequently underestimated in such studies (meaning that the transverse velocity from proper motion was correspondingly underestimated). 


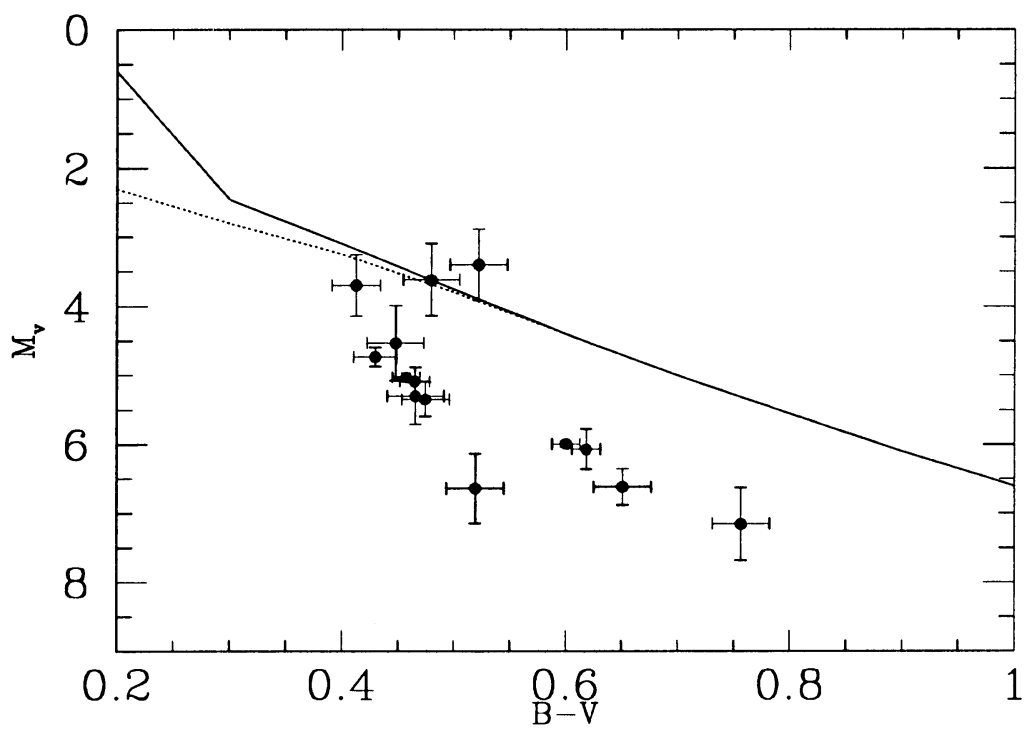

Figure 2. Position in the CMD of the subdwarfs with a $[\mathrm{Fe} / \mathrm{H}]$ between -2.4 and -2.0 dex. The line is the Hyades sequence (observed - line, zero-age - dots). For the first time, we are able to see the turnoff and subgiant region in field subdwarfs, almost as clearly as in globular clusters !

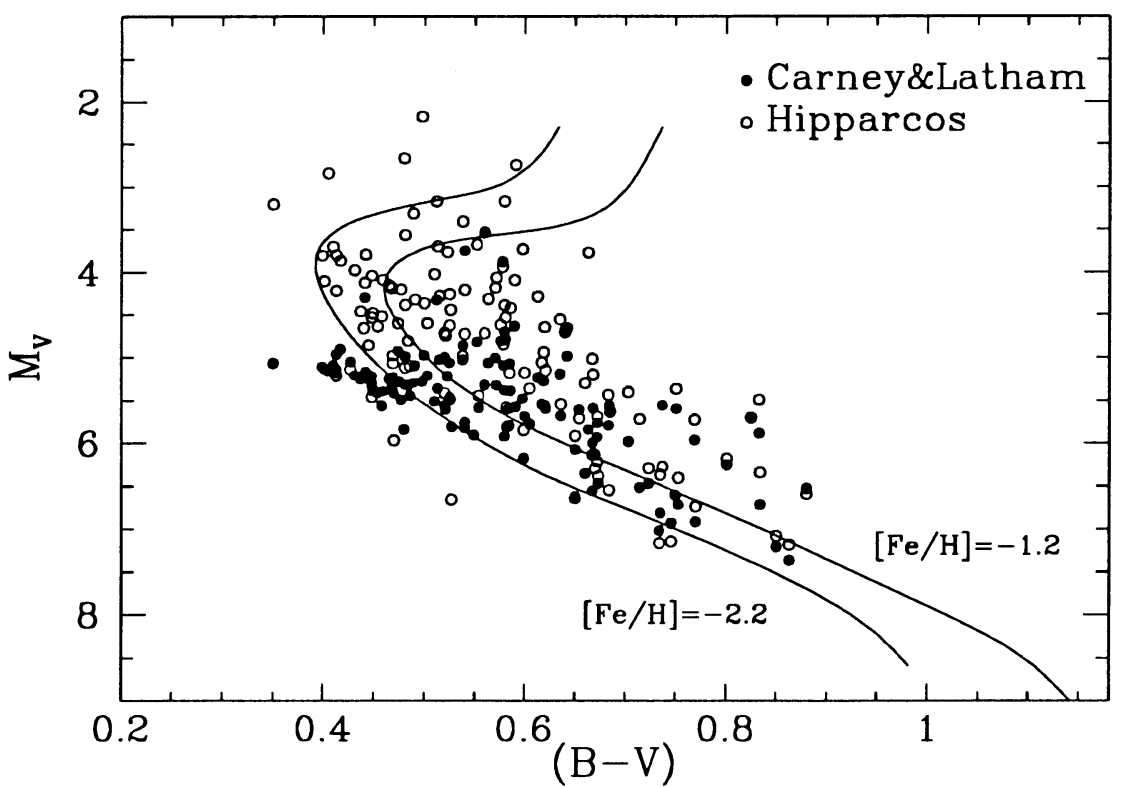

Figure 3. Comparison of the position in the CMD for some stars common to the C94 programme and to Hipparcos. $15 \mathrm{Gyr}$ models isochrones from d'Antona et al. (1996) are superimposed. The unsuspected high number of evolved early type subdwarfs is clear. 


\section{Metal-deficient ZAMS}

The displacement of the ZAMS in the CMD for various metallicities can be calibrated directly using the subdwarfs. The calibrators must be unevolved, and detected or suspected binaries, as well as objets with an uncertain parallax or large colour excess, are excluded. This reduces considerably the number of suitable calibrators. We shall increase this number by gathering more metallicity measurements, while refining the CORAVEL $[\mathrm{Fe} / \mathrm{H}]$ calibration for $\mathrm{G}$ and deficient $\mathrm{F}$ stars.

\section{Globular Cluster distances}

The great usefulness of subdwarf luminosity calibrations is that the Globular Cluster (GC) distances can then be directly determined via mainsequence fitting to the subdwarf sequences of same $[\mathrm{Fe} / \mathrm{H}]$. No theoretical prediction is needed, except the assumption that field and GC stars of the same metallicity are similar. The reddening and metallicity of GCs must be known independently.

\section{Globular Cluster ages}

Recently, a considerable effort has been put into acquiring good CMD for Globular Clusters down to the lower main sequence. From these and the Hipparcos subdwarfs, reliable distances can be obtained. Then the turnoff luminosity, which is known to be a very robust prediction of stellar evolution and a sensitive function of age, can be compared directly to the model predictions, without any assumption on the poorly known colour and temperature calibrations. In practice, to avoid the uncertainty caused by the vertical tangent at the turnoff, we use the magnitude of the two points 0.04 mag redder than the turnoff.

The effect of errors on the reddening or metallicity of the clusters on this method is relatively severe $\left(\Delta a g e[\mathrm{Gyr}] \simeq E(B-V) \cdot 10^{+2}[\mathrm{mag}], \Delta a g e[\mathrm{Gyr}] \simeq\right.$ $12 \cdot \Delta[\mathrm{Fe} / \mathrm{H}])$. For relative age estimations, indirect comparative methods are to be preferred.

Absolute ages, however, are another matter, and the subdwarf method is the most accurate. Pending some remaining uncertainties on stellar evolution models, the average ages of the oldest GC provided by this method can be considered as a very tight constraint on the lower limit of the age of the Universe.

Once good distances are known for a sample of GC, the position of other indicators can be calibrated, such as the $\mathrm{HB}$ luminosity, or the $[\mathrm{Fe} / \mathrm{H}]$ dependence of RR Lyrae luminosity. 
The subdwarf calibration will also permit a direct evaluation of the age of field subdwarfs relative to the globular clusters, a possible clue to the formation history of the Galaxy.

\section{Towards reliable globular cluster ages}

As more subdwarf CORAVEL measurements get added to the sample, the $[\mathrm{Fe} / \mathrm{H}]$ calibration will be refined, and the number of suitable unevolved calibrators will increase. Once precise subdwarf sequences are determined, we shall try, in collaboration with stellar evolution modelists, to explore the changes needed in the models to fit the position and slope of the observed sequences, thereby improving the turnoff luminosity predictions. Finally, the effect of diffusion on the ages predicted by the models will be studied. The results will be presented in the Hipparcos meeting in Venice (May 1997).

\section{References}

Carney B.W., Latham D.W., Laird J.B. et al. 1994, AJ 107, 2240 (C94)

d'Antona F., Mazzitelli I., 1996, ApJ 456, 329

Edvardsson B., Andersen J., Gustafsson B. et al., 1993, A\&A 275, 101 (E93)

Mayor M., 1980, A\&A 87, L1

Ryan S.G., Norris J.E., 1991, AJ 101, 1835

Zinn R., West M.J., 1984, ApJSS 55, 45 